\title{
Longitudinal variation of the pickup-proton-injection efficiency and rate at the heliospheric termination shock
}

\author{
H. J. Fahr ${ }^{1}$, K. Scherer ${ }^{2}$, M. S. Potgieter ${ }^{3}$, and S. E. S. Ferreira ${ }^{3}$ \\ 1 Argelander Institut für Astronomie, Universität Bonn, Germany \\ e-mail: hfahr@astro.uni-bonn.de \\ 2 Institut für Theoretische Physik IV, Ruhr Universität Bochum, 44780 Bochum, Germany \\ e-mail: kls@tp4.rub.de \\ 3 Unit for Space Physics, North-West University 2520, Potchefstroom, South Africa \\ e-mail: [Stefan.Ferreira;Marius.Potgieter]@nwu.ac.za
}

Received 12 February 2008 / Accepted 27 May 2008

\section{ABSTRACT}

\begin{abstract}
Context. Pickup ions are considered to be the seed population of the anomalous cosmic rays. The acceleration of the pickup ions at the solar wind termination shock depends on the efficiency with which they are injected into the first reflection mode, from where acceleration into high energy anomalous cosmic rays begins. In this letter, we study the longitudinal variation of this efficiency.

Aims. We consider the specific MHD conditions at the longitudinal position of the termination shock, which are highly sensitive to the local inclination angle between the magnetic field and the shock normal. We determine the probability for pickup-ions to become reflected which depends on how the inclination angle varies with longitude. This is the first and relevant step in the acceleration of pickup ions to anomalous cosmic rays.

Methods. The most dramatic change in the magnetic tilt angle occurs in the current sheet between two consecutive solar wind sector structures of different polarity, when they pass over the shock. We consider this effect by modeling the longitudinal variation in the injection efficiency. Starting from calculations of the pickup-ion reflectance probability as a function of the magnetic tilt angle with respect to the normal, we find an analytic expression for the injection efficiency: to do this we need the magnetic tilt angle as a function of longitude, which we obtain from an analytic representation of the termination shock shape. This enables us to determine the longitudinal dependence of the injection efficiency.

Results. We find that a substantial variation in the injection efficiency of a factor of 3 occurs when passing from the upwind part to the flanks of the termination shock. Folding this with the longitudinal dependence of the pickup ion density gives an analytic expression the injection rate which varieis by a factor of 2 between up- and ecliptical crosswind direction and a factor of 5 between cross- and downwind direction.

Conclusions. Our model indicates that injection into the anomalous cosmic ray acceleration predominantly occurs in the ecliptical crosswind direction e.g. in the flanks of the termination shock.
\end{abstract}

Key words. solar system: general - shock waves - magnetohydrodynamics (MHD) - ISM: cosmic rays - acceleration of particles

\section{Introduction}

Neutral interstellar hydrogen passes over the interface structure of the heliosphere and enters the inner heliosphere, i.e. the supersonic solar wind regime. On ionization, they are converted into newly born ions, called pickup ions (PUIs), which are convected outward with the solar wind. While they are comoving with the solar wind, they are subjected to other processes such as adiabatic cooling and momentum diffusion, and should be described by a phase space transport equation. A solution of this transport equation was given by Chalov \& Fahr (1996, 2000). They showed, that most PUIs are swept over the termination shock (TS) and comove with the subsonic solar wind downstream. Nevertheless, a few of them, due to special conditions in phase space, are reflected and start to undergo the acceleration process to become anomalous cosmic rays (ACRs), i.e. are injected into the ACR energy regime. These special conditions are caused by adiabatic reflections in condensed (downstream) magnetic fields: i.e. they are connected with low ion velocities relative to the shock, which are valid for ions sitting in the sunward hemisphere of the shell-like distribution in the upstream wind frame. The first reflection via pitch-angle scattering brings the reflected ions into an extended velocity shell of the ion population comoving with the upstream solar wind. This behavior of reflected PUIs is described by the above mentioned authors as a PUI reflectance efficiency, which is strongly sensitive to the magnetic tilt angle $\psi$ between the magnetic field and the shock normal. To avoid confusion we note, that with the tilt angle of the heliospheric current sheet, we refer to the angle between the magnetic field vector and the normal, as the magnetic tilt angle.

In the following we apply these results and develop an efficiency depending on the ecliptical longitude of the TS. We represent the shape of the TS by an ellipse, with one focus at the Sun and with upwind and downwind locations taken from the model by Scherer \& Ferreira (2005). The magnetic field in the inner heliosphere upstream of the TS is calculated using the Parker field, allowing an analytic representation of the magnetic tilt angle $\psi$ against longitude $\varphi$. A complication has also to be taken into account, which is the passage of the heliospheric magnetic field sector over the TS. While a sector structure passes over the TS, the magnetic field vector rotates by about 180 degrees, which 


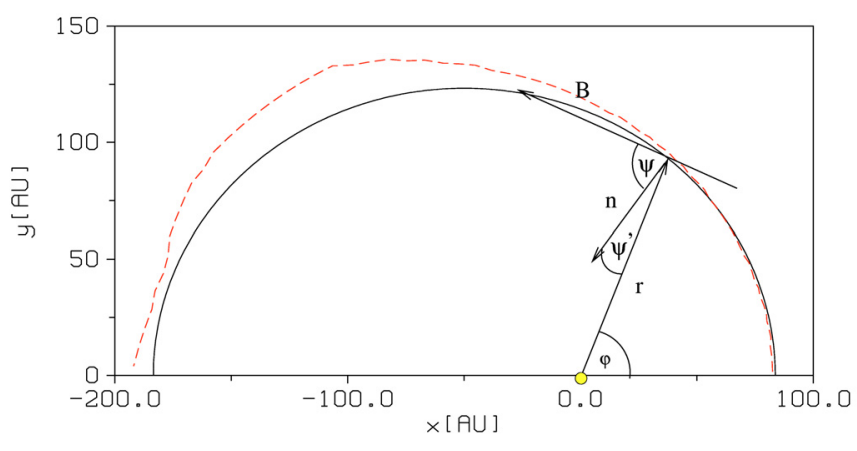

Fig. 1. The assumed ellipse for the TS (black line) and the TS determined from the hydrodynamic model (red line) by Scherer \& Ferreira (2005) are shown. In addition the radius vector $\boldsymbol{r}$, the magnetic field vector $\boldsymbol{B}$ and the normal vector $\boldsymbol{n}$ are sketched, including the longitudinal angle $\varphi$, the magnetic tilt angle between the magnetic field, the normal $\psi$, and its co-angle $\psi^{\prime}$.

strongly influences the magnetic tilt angle $\psi$ and therefore the injection efficiency $E$.

Approximating the results of Chalov \& Fahr (1996), we are finally able to derive an analytic expression for the injection efficiency and connect with it the injection rate.

In Sect. 1 we will describe the geometry of the TS and its analytic representation as well as the magnetic tilt angle $\psi$. Continuing in Sect. 2 with an analytic representation of the magnetic tilt angle $\psi$ dependence on the injection efficiency $E$, we are able to estimate the longitudinal dependence of the injection efficiency $E(\psi(\varphi))$, which is inverse fraction of all pick-up ions running into the shock and to the number of those reflected back into the upstream direction.

\section{Geometry of the termination shock and the magnetic tilt angles}

The termination shock can be approximated by an ellipse, of which one focus is the Sun. With the help of the aphelion distance, which is given by the tail TS distance (185 AU) and the perihelion distance given by the upwind TS distance (82 AU), the relevant parameters of the ellipse, i.e. the eccentricity $\varepsilon$ and the major axis $a$, can be calculated from the standard representation of its radius:

$r:=\frac{a\left(1-\varepsilon^{2}\right)}{1+\varepsilon \cos \varphi}$

where $r$ and $\varphi$ are the radius and the true anomaly $\varphi$, respectively in our case the true anomaly is identical to the longitudinal angle: we therefore select the coordinate system, for which the nose direction coincides with $0^{\circ}$ and the tail with $180^{\circ}$ longitude. The nose and tail distances of the TS are taken from the hydrodynamic model by Scherer \& Ferreira (2005). Applying Eq. (1) we can easily determine the semi-major axis ( $a=133.5 \mathrm{AU})$ and the eccentricity $(\varepsilon=0.385)$. In Fig. 1 this ellipse and the TS shape determined by the hydrodynamic model are shown. As can be seen, the deviations from the model calculations are sufficiently small for our purposes. In addition, we indicate the radius vector $\boldsymbol{r}$, the magnetic field vector $\boldsymbol{B}$, and the normal vector $\boldsymbol{n}$ including the longitudinal angle $\varphi$, the magnetic tilt angle between the magnetic field, and the normal $\psi$ and its co-angle $\psi^{\prime}$.

The magnetic field in the local interstellar medium (LISM) may influence the outer boundaries of the heliosphere, especially the shape and distance of bow shock and the heliopause (Ratkiewicz et al. 1998; Pogorelov \& Matsuda 1998;
Alexashov \& Izmodenov 2005; Ratkiewicz 2006; Opher et al. 2007; Pogorelov et al. 2008). This possible LISM magnetic pressures mainly influence the distance to the termination shock, but much less its geometry, and thus should only very mildly change the longitudinal variation in the injection efficiency presented in this paper.

Moreover, our approach fits to the solar minimum conditions, when the magnetic current sheet is close to the ecliptic. Under solar maximum conditions with current sheets extending to moderate latitudes of about 30 to 60 degrees the consideration of latitudinal injection variations would be needed. We discuss this extended view together with the latitudinal dependence in a forthcoming paper.

We can easily derive the slope of the tangent at a point $(r, \phi)$ using:

$\frac{\mathrm{d} r}{\mathrm{~d} \varphi}=a\left(1-\varepsilon^{2}\right) \frac{\varepsilon \sin \varphi}{(1+\varepsilon \cos \varphi)^{2}}$.

Applying Eq. (2) we find, that the components of the tangent vector are:

$t_{r}=\mathrm{d} r$

$t_{\varphi}=r \mathrm{~d} \varphi=\frac{r \mathrm{~d} r}{a\left(1-\varepsilon^{2}\right)} \frac{(1+\varepsilon \cos \varphi)^{2}}{\varepsilon \sin \varphi}=\frac{1+\varepsilon \cos \varphi}{\varepsilon \sin \varphi} \mathrm{d} r$.

The components of the normal vector $\boldsymbol{n}$ are then $n_{r}=-t_{\varphi}$ and $n_{\varphi}=t_{r}$. To demonstrate the longitudinal dependence of the injection rate, it is sufficient to describe the heliospheric magnetic field (HMF) applying Parker's model, which in the ecliptic and at large distances close to the TS this magnetic field model has only an azimuthal component, which either is positive or negative depending on the polarity of the source field at the solar surface. This implies that the HMF is perpendicular to the radius vector $\boldsymbol{r}$ and its inclination with respect to the normal is $\psi^{\prime}=90^{\circ}-\psi$, where the cosine of this angle is given by:

$\cos \psi^{\prime}=\left|\frac{\boldsymbol{r} \cdot \boldsymbol{n}}{n r}\right|=\frac{1+\varepsilon \cos \varphi}{\sqrt{1+2 \varepsilon \cos \varphi+\varepsilon^{2}}}$.

Thus, the angle $\psi$ is given by $\psi=\operatorname{asin}\left(\cos \psi^{\prime}\right)$, as shown in Fig. 2. Our results could be compared with those presented earlier by Chalov \& Fahr (1996), Chalov et al. (1997), Chalov \& Fahr (2000) and Chalov (2005).

\section{The dependence of efficiency function on sector-structure and longitude}

As found by Kucharek \& Scholer (1995) and Chalov \& Fahr (1996), the injection efficiency $E$ for protons is highly sensitive to the magnetic tilt angle $\psi$, which itself is a function of longitude (see Fig. 2 and Eq. (4)). The sensitivity function as discussed in Chalov \& Fahr (1996), for average turbulence levels i.e. $\left\langle\delta B^{2} / B^{2}\right\rangle=0.1$ (where $\delta B$ is the magnetic field fluctuation), is fitted by:

$E(\psi)=E_{0} \exp \left(-k \psi^{n}\right)$

where $\psi$ is measured in degrees, $k=2.08 \times 10^{-20}$ and $n=10.9$, taken from the curve calculated for the average turbulence levels in Chalov \& Fahr (1996): clearly this shows a constant behaviour for $\psi<65^{\circ}$ and a strong fall-off beyond $\psi>65^{\circ}$ (see Fig. 3). The initial injection efficiency $E_{0}$ takes values between 0.2 to 0.6 , depending on the turbulence levels (Chalov \& Fahr 1996). To avoid this uncertainty in $E_{0}$ we use in what follows injection efficiency normalized to $E_{0}$. During passages of magnetic sector 


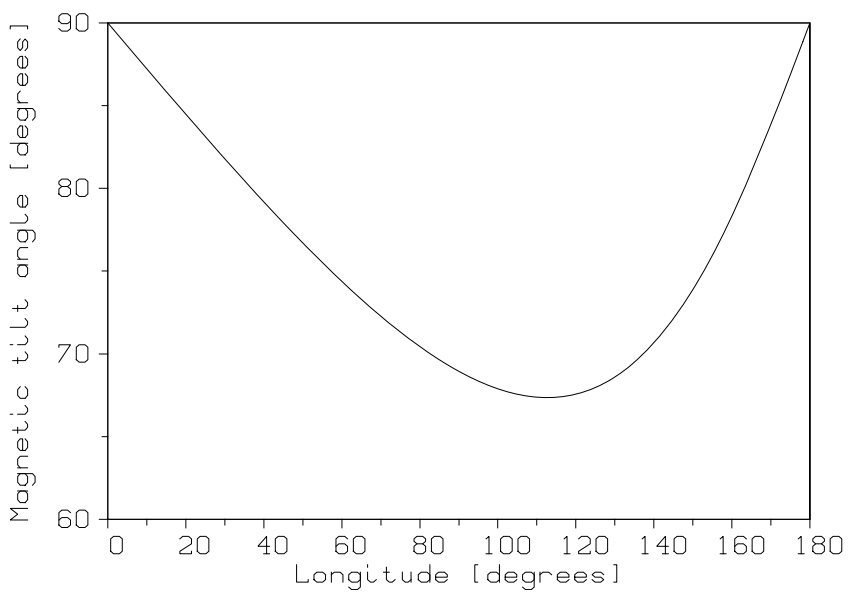

Fig. 2. The magnetic tilt angle $\psi$ between the magnetic field and the normal vector is shown as a function of longitude.

structures over the TS, the magnetic tilt angle $\psi$ varies dramatically. Therefore, we need to take into account what effect this has on the injection efficiency.

We restrict the model to the ecliptic and to solar minimum to maintain a clearly defined sector structure. At the position of the TS, it is evident that the magnetic field vector, as described by Parker's model, is perpendicular to the radius vector $\boldsymbol{r}$. At passages of sector structures, the polarity of the field changes roughly in one day, implying that the magnetic field vector rotates by $180^{\circ}$ and $\psi$ changes accordingly. During this event, there are periods in which the field orientation with respect to the normal of the TS is very favorable for high injection efficiencies. This was recognized earlier by Kucharek \& Scholer (1995) and Smith (1993).

According to Voyager data (Burlaga et al. 2003), a sector structure persist approximately between 2 and 10 days. This is the persistence time $T$, while the change to the other polarity occurs in one day. During this day, the magnetic tilt angles $<70^{\circ}$ persists for $140 / 180 \%$ of that day, e.g. about $80 \%$ of that period are favorable to injection. As soon as magnetic tilt angles below $70^{\circ}$ are realized, the injection efficiency is almost constant at $E_{0}$. Thus dividing this day of change into an efficient fraction $\tau_{\mathrm{e}}$ and an inefficient one $\tau_{\mathrm{i}}$, including the persistence time $T$, we express the mean injection efficiency $\tilde{E}$

$\tilde{E}=\frac{\left(T+\tau_{\mathrm{i}}\right) E(\psi)+\tau_{\mathrm{e}} E_{0}}{T+\tau_{\mathrm{e}}+\tau_{\mathrm{i}}}$.

As described above, $\tau_{\mathrm{e}} \approx 0.8$ and $\tau_{\mathrm{i}} \approx 0.2$. In Fig. 4 , we display this function for three different persistence times $T$, e.g. 2 days (red dashed-dotted), 5 days (cyan dashed-dotted), and 10 days (blue dashed-dotted). The black dashed line is the reference curve without magnetic sector structure passing by, showing how low the injection efficiency would be at longitude $0^{\circ}$ and $180^{\circ}$ without time-variable magnetic fields.

The surface injection rate is given by the product of the injection efficiency, the number density of the PUIs and the solar wind $V_{\text {sw }}$ speed. We fit the PUI-flux $\Phi_{\text {PUI }}(\varphi)=n_{\mathrm{PUI}}(\varphi) V_{\mathrm{sw}}(\varphi)$ normalized to the flux level of the TS with a straight line $\Phi^{\prime} \varphi=$ $\left.\Phi_{\mathrm{PUI}}(\varphi) / \Phi_{\mathrm{PUI}}(0)=A \cdot \varphi+B\right)$, according to that discussed for solar minimum by Scherer et al. (2006), with $A=-3.9 \times 10^{-3}$ and $B=1$, i.e. $\Phi^{\prime}\left(0^{\circ}\right)=1$ and $\Phi^{\prime}\left(180^{\circ}\right)=0.3$. Thus, the normalized injection rates, $\beta$, is the product of the normalized injection efficiency and the normalized PUI flux. which are shown in Fig. 4 as solid lines, where the colors correspond to the different cases discussed for the normalized injection efficiency above.

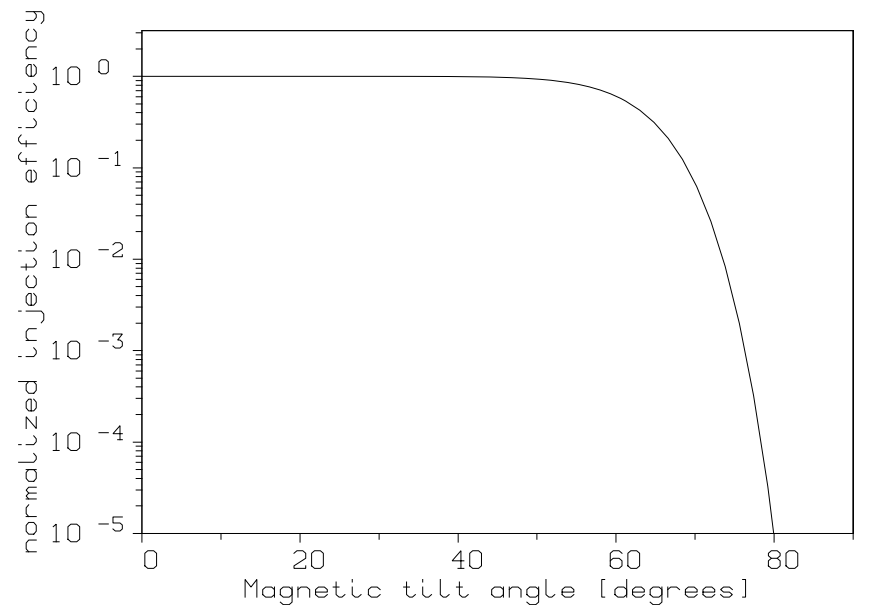

Fig. 3. The normalized injection efficiency as a function of magnetic tilt angle.

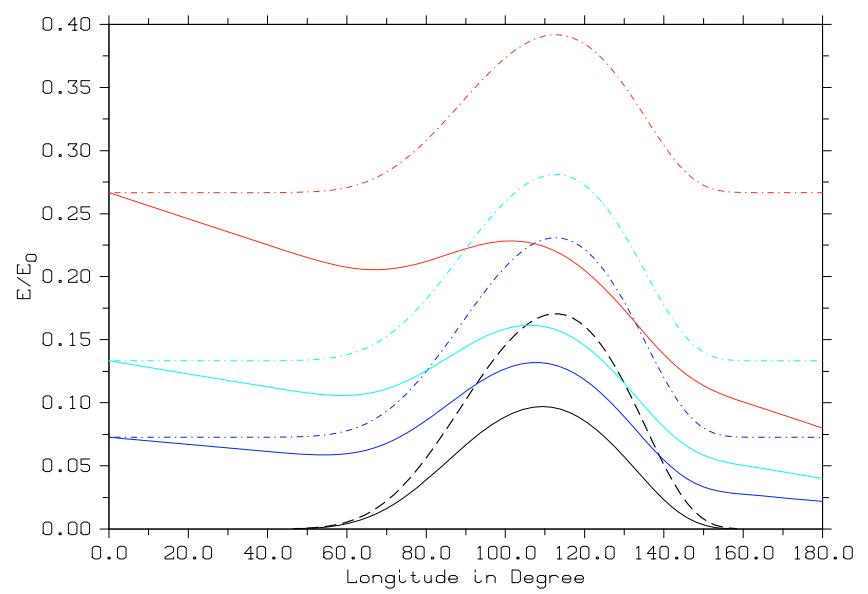

Fig. 4. The normalized injection efficiency as modeled above for different persistence times, 2 days (red dashed-dotted), 5 days (cyan dasheddotted), and 10 days (blue dashed-dotted) and the reference curve without sweeping sector structures. The solid lines represent the injection rate, and the colors correspond to those of the injection efficiency. The injection rate is the multiplication of the normalized injection efficiency with the normalized PUI number density. The dotted lines indicate the position of Voyager 1 (V1) and 2 (V2), respectively.

While the injection efficiencies vary with longitude by a factor $\approx 3$, between upwind and crosswind directions, the corresponding injection rates vary only by factor $\approx 2$. This reveals that the injection rate in the flanks (crosswind) is larger than in the nose, and hence we can expect higher ACR-flux in the flanks, compared to that at the nose.

\section{Discussion}

It was first proposed by Chalov (1993), that the injection efficiency could increase towards the flanks. This author pointed out qualitatively that conditions for the injection of PUIs improve with increasing longitude because of smaller magnetic tilt angles between the magnetic field and shock surface normal in the flanks. It was argued that PUI's had to achieve a critical velocity before they could be reflected by the potential. This condition for unaccelerated PUIs was only fulfilled at longitudes higher than $\approx \varphi=90^{\circ}$. No theoretical value of the resulting injection efficiency could be worked out from this approach (see also for 
a more detailed discussion Chalov \& Fahr 1996; Chalov et al. 1997; Chalov \& Fahr 2000; Chalov 2005).

Later using shock simulations studies, Kucharek \& Scholer (1995) found a dependence of the injection efficiency on magnetic tilt angle. In this line Zank et al. (1996) carried out a single particle reflection studies at a quasi perpendicular shocks and found some criteria for reflected PUIs to enter into the ACR acceleration regime. Their derivation was based on shell distribution functions and an analytic expression for injection efficiency in the case of perpendicular shocks.

Schwadron \& McComas (2003) developed further arguments why this should be true. They discussed Favored Acceleration Locations at the TS (FALTS) connected with the magnetic field geometry between fast and slow solar wind structures, induced by moving the magnetic footpoints at the solar surface. McComas \& Schwadron (2006) discussed a different reason why, at the nose of the heliospheric TS, the injection efficiency is small, increasing towards the flanks; this is caused by the magnetic field lines sweeping in and out over a blunt TS. Furthermore, our analytic approach does not simulate a blunt TS, since almost all models (see Müller et al. 2008, for a comparison of such models) are unable to describe such a blunt-shaped TS geometry. To describe the injection efficiency, we model the merging sector structure, instead of a meandering of the magnetic fields across the shock as in the case of a blunt TS.

Most the above mentioned attempts, apart from Kucharek \& Scholer (1995), provide only qualitative evidence that the injection efficiency is dependent on longitudinal, while our approach attemps to determine the injection efficiency quantitatively. The absolute value of the injection efficiency was kept as a parameter in the past, ranging from values of 0.0003 to 0.9 (see le Roux \& Fichtner 1997). However, large values of the injection efficiency may become critical in view of the physics of the ACR modulated shock (Chalov \& Fahr 1995). If the TS converts low energetic ions into ACR's too efficiently and, therefore, loses too much energy from the background plasma, then the shock becomes quasi-isothermal and collapses towards a shock-free deceleration case with a compression ratio of 1 . In view of the above mentioned uncertainties it had become necessary to calculate the injection efficiency more quantativly, as attemped in this paper.

\section{Conclusions and outlook}

We have demonstrated that the PUI-injection rate varies with longitude by a factor of $\approx 2$. The lowest values are in the nose $\left(0^{\circ}\right)$ direction (upwind), whereas the highest values are in (ecliptical) crosswind directions (around $100^{\circ}$ ), decreasing towards lower values in the tail $\left(180^{\circ}\right)$ direction (downwind) as compared to those upwind. Depending on the persistence time of the sector structure, the injection efficiency at the nose is much higher than expected in the absenence of sector structure passages. For the cases discussed here, the normalized injection efficiency varies from 0.07 ( 10 day persistency) to 0.27 ( 2 day persistency) at the nose, while without sector structure passages over TS, the upwind injection efficiency is approximately $10^{-6}$ and thus negligible. The upper and lower curves for both the normalized injection efficiencies and rates can be understood as the envelopes of the most probable values, which we treat as a curve describing the 5 day persistence of a sector structure.
The favourite injection places are the flanks, for which even the normalized injection efficiency without sector structure is approximately 0.16 , while those with sectoring are slightly higher (0.2 to 0.4 ). Finally, the injection rates show a similar behavior compared to the normalized injection efficiency, where both values, at the nose, coincide because of the normalizations, but, at the flanks, the corresponding normalized injection efficiencies are higher by a factor of 2 .

Comparing our representation of the TS by an ellipse with the calculated model TS (see Fig. 1), it is evident that smaller inclination angles $\psi$ in the modeled TS would produce even higher normalized injection efficiency in the flanks.

The Voyager 1 and 2 spacecraft are located at positions of $34^{\circ}$ and $-29.6^{\circ}$ in ecliptic latitude as well as $253^{\circ}$ and $288^{\circ}$ in ecliptic longitude, respectively. The interstellar wind comes from a direction of $-7^{\circ}$ ecliptic latitude and $277^{\circ}$ ecliptic longitude. Transforming the Voyager positions into the wind coordinate system, we derive for the longitudes of $24^{\circ}$ and $-11^{\circ}$, respectively. The latter corresponds, because of our axis symmetry, to $+11^{\circ}$ longitude in the wind system. Thus, we would expect to observe a lower flux of ACRs at the positions of Voyager 1 and 2 spacecraft (see the dotted line in Fig. 4) compared to flux expected at the flanks. This effect should be modulated by the latitudinal variation of the normalized injection rate, which we propose to a future paper. This require the developement of a three dimensional perspective of injection efficiency. Moreover, we propose to find a solution for solar maximum conditions. This study can also be extended to other PUI species.

Acknowledgements. K.S. and H.F. acknowledge support by the Deutsche Forschungsgemeinschaft (DFG) in the frame of the project FI 706/6-2 "Heliocauses" carried out within the framework of the DFG priority program 1176 within CAWSES and Fa97/31-2 "Magnetokinetics" M.S.P. and S.E.S.F. are grateful for financial support granted by the South African National Research Foundation.

\section{References}

Alexashov, D., \& Izmodenov, V. V. 2005, A\&A, 439, 1171

Burlaga, L. F., Ness, N. F., \& Richardson, J. D. 2003, J. Geophys. Res., 108, 8028

Chalov, S. V. 1993, Planet. Space Sci., 41, 133

Chalov, S. V. 2005, Adv. Space Res., 35, 2106

Chalov, S. V., \& Fahr, H. J. 1995, Planet. Space Sci., 43, 1035

Chalov, S. V., \& Fahr, H. J. 1996, Sol. Phys., 168, 389

Chalov, S. V., \& Fahr, H. J. 2000, A\&A, 360, 381

Chalov, S. V., Fahr, H. J., \& Izmodenov, V. 1997, A\&A, 320, 659

Kucharek, H., \& Scholer, M. 1995, J. Geophys. Res., 100, 1745

le Roux, J. A., \& Fichtner, H. 1997, ApJ, 477, L115

McComas, D. J., \& Schwadron, N. A. 2006, Geophys. Res. Lett., 33, 4102

Müller, H., Florinski, V., Heerikhuisen, J., et al. 2008, A\&A, in press

Opher, M., Stone, E. C., \& Gombosi, T. I. 2007, Science, 316, 875

Pogorelov, N. V., \& Matsuda, T. 1998, J. Geophys. Res., 103, 237

Pogorelov, N. V., Heerikhuisen, J., \& Zank, G. P. 2008, ApJ, 675, L41

Ratkiewicz, R. 2006, Astrophysics and Space Sciences Transactions, 2, 11

Ratkiewicz, R., Barnes, A., Molvik, G. A., et al. 1998, A\&A, 335, 363

Scherer, K., \& Ferreira, S. E. S. 2005, Astrophys. Space Sci. Trans., 1, 17

Scherer, K., Ferreira, S. E. S., Potgieter, M. S., \& Fichtner, H. 2006, in Physics of the Inner Heliosheath, 5th Annual IGPP Int. Astrophys. Conf., ed. J. Heerikhuisen, V. Florinski, G. Zank, \& P. Pogorelov, 858, 20

Schwadron, N. A., \& McComas, D. J. 2003, Geophys. Res. Lett., 30, 41

Smith, E. J. 1993, Advances in Space Research, 13, 5

Zank, G. P., Matthaeus, W. H., \& Smith, C. W. 1996, J. Geophys. Res., 101, 17093 